\title{
Adult Laryngeal Hemangioma - A Rare Case Report
}

IHK Chen ${ }^{a, b}$, Abdul Jalal SY ${ }^{a}$, Singh $B^{a}$, Mat Baki $M^{b}$

${ }^{a}$ Department of Otorhinolaryngology-Head and Neck Surgery, Putrajaya Hospital

${ }^{b}$ Department of Otorhinolaryngology Head \& Neck Surgery, Universiti Kebangsaan Malaysia Medical Centre, Kuala Lumpur, Malaysia

\section{ABSTRACT}

Laryngeal hemangiomas are classified into an adult and infantile forms. The incidence of laryngeal hemangioma in adults is rare. While infantile hemangiomas are more frequently found in subglottic regions and regress with age, adult hemangiomas occur commonly in supraglottic and glottic regions and they do not regress spontaneously. In this article, we report a case of laryngeal cavernous hemangioma in a 51-year-old male who was treated successfully with endolaryngeal microsurgical technique without any recurrence through one year follow up.

KEYWORDS: Hemangioma, Cavernous Hemangioma, Larynx, Adult, Glottis

\section{INTRODUCTION}

Benign neoplasms of the larynx are infrequent and occur in two main forms: adult and infantile hemangiomas. Hemangioma is the commonest congenital benign tumor and defined as vascular malformation consisting of abnormal and dilated vessels. It is a slow progressing vascular tumor, which is self-limiting. It is commonly found in children and rare in adults. The incidence of infantile laryngeal hemangioma is 4-5 percent, however, the incidence in adult remains unclear due to the paucity of case reports. ${ }^{1}$ While infantile hemangioma resolves in nearly half by five years of age and further regress with age, adult hemangiomas do not regress spontaneously. ${ }^{2}$ In adults, they are commonly found in males and eighty percent of these lesions lie in the supraglottis, followed by glottis and subglottis. ${ }^{3}$

Laryngeal hemangiomas are generally remained silent over a period of time and do not warrant any treatment until obstructive symptoms appear. The presenting symptoms differ depending on the location of the lesion: dysphagia, hoarseness, and shortness of breath are more commonly seen in adults; whereas stridor and respiratory distress are the main symptoms in infantile hemangioma.

Corresponding Author:

Prof. Dr. Marina Mat Baki

Department of Otorhinolaryngology-Head and Neck Surgery, National University of Malaysia (PPUKM), Jalan Yaakob Latif, Bandar Tun Razak, 56000 Batu 9 Cheras, Wilayah Persekutuan Kuala Lumpur, Malaysia

Tel No : +6019-6534308

Email : marinamatbaki@ppukm.ukm.edu.my
Cases with large laryngeal hemangiomas with obstructive symptoms require treatment. To the best of our knowledge, only one adult glottic hemangioma of South East Asia was reported in English literature where complete excision of the hemangioma was performed using endolaryngeal microsurgery (ELMS) technique. ${ }^{4}$ This is the second similar case to be reported using the same technique allowing complete resection of the tumour in a 51-year-old male presented with obstructive symptoms who is now disease-free through one year follow up.

\section{CASE REPORT}

A healthy 51-year-old male presented to the Department of Otorhinolaryngology Putrajaya Hospital with an acute onset of difficulty of breathing with blood in sputum. He had hoarseness for one year, which is progressively worsening for the past six months along with globus sensation. He denied dysphagia, noisy breathing, and aspiration symptoms. He was a chronic smoker of 30 pack years. There was no history of chronic cough, reflux symptoms, voice abuse, or alcohol consumption.

Upon examination, there was no audible stridor and a flexible nasopharyngeal laryngoscope revealed an irregular red-purplish polypoidal lesion over the anterior two-thirds of the left vocal cord, obstructing thirty percent of the airway. Bilateral vocal cords were mobile during phonation and respiration. Neck examination showed no cervical lymphadenopathy and laryngeal crepitus was present with an intact laryngeal framework. 
Correlating these features of a laryngeal lesion with an associated history of smoking and age, a differential diagnosis of malignant neoplasm was arrived at. The diagnostic direct laryngoscope was performed under general anesthesia revealed an irregular red-purplish polypoidal lesion with a smooth mucosal surface and pedunculated base arising from the anterior two-thirds of the left vocal cord (Figure 1). The anterior commissure of the glottis and the right vocal cord was free of tumour. The supraglottic and subglottic regions were also normal. The biopsy was taken from the lesion and there was minimal bleeding where hemostasis was secured using adrenaline-soaked ribbon gauze. The initial histopathology examination (HPE) results revealed benign features with inflamed laryngeal nodule and there was no evidence of atypia, dysplasia, or malignancy.

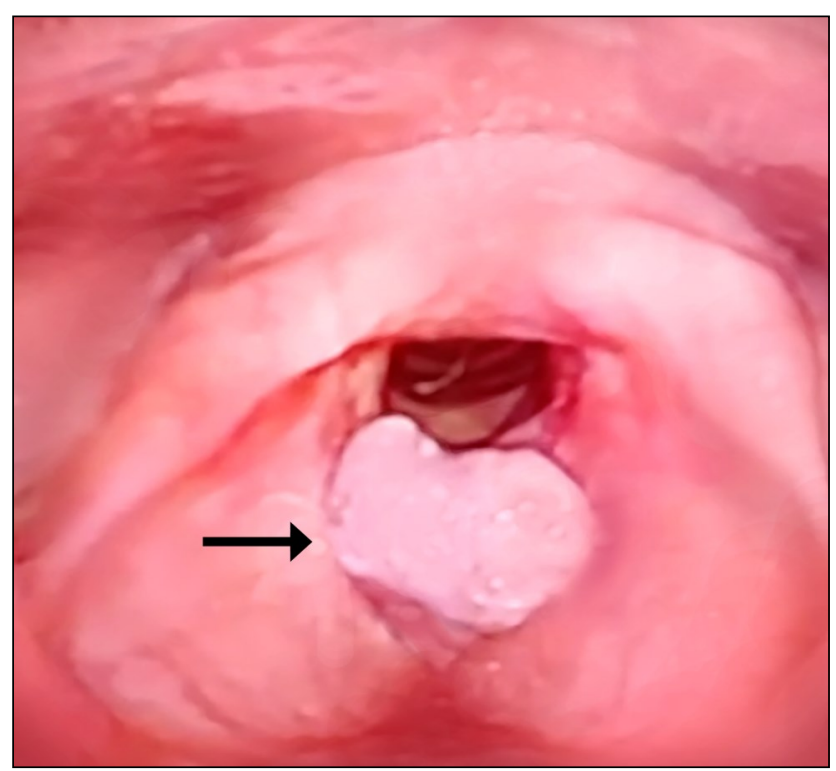

Figure 1: Endoscopic view of an irregular red-purplish polypoidal lesion (arrow) with smooth mucosal surface and pedunculated base arising from the anterior two-thirds of the left vocal cord.

The patient had then undergone ELMS for excision of the tumor while preserving voice. A polypoidal pink-purplish mass with a pedunculated base was excised in toto from the left vocal cord measuring approximately 8 millimeters $(\mathrm{mm})$ using a simple superficial excision technique. The mass was lifted and pulled to the contralateral side with the aid of laryngeal right turned cupped forcep and the narrow pedunculated base was cut using laryngeal straight scissor. The mass was friable and bled on touch where haemostasis was secured with adrenalinesoaked ribbon gauze. The final HPE results revealed that the polypoidal lesion was covered by stratified squamous epithelium and the stroma composed of many interconnecting large vascular channels lined by flat endothelial cells with blood present in the vascular channels (Figure 2). A diagnosis of cavernous hemangioma of glottic was confirmed.

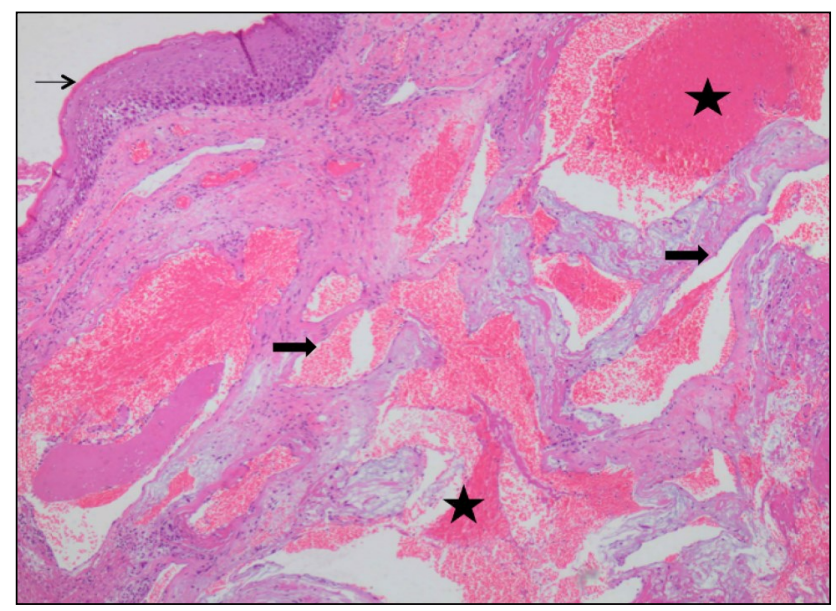

Figure 2: Histological appearance of cavernous laryngeal hemangioma. The lesion is covered by stratified squamous epithelium (arrow). The stroma composed of many interconnecting large vascular channels lined by flat endothelial cells (thick arrow) and fibrocartilagenous tissue with areas of fibrinoid necrosis. Blood present in the vascular channels (star).

In postoperative assessment, the patient's voice has returned to near normal a week post-procedure. His voice handicap index-10 (VHI-10) was within the normal range (total score, 5), GRBAS scale (Grade, Roughness, Breathiness, Asthenia, Strain) showed slight deviance in grade and roughness and maximum phonation time was 18 seconds. The repeated flexible nasopharyngeal laryngoscope was unremarkable (Figure 3). The patient was then given follow up in our clinic three monthly for surveillance. He is currently disease-free through one year follow up.

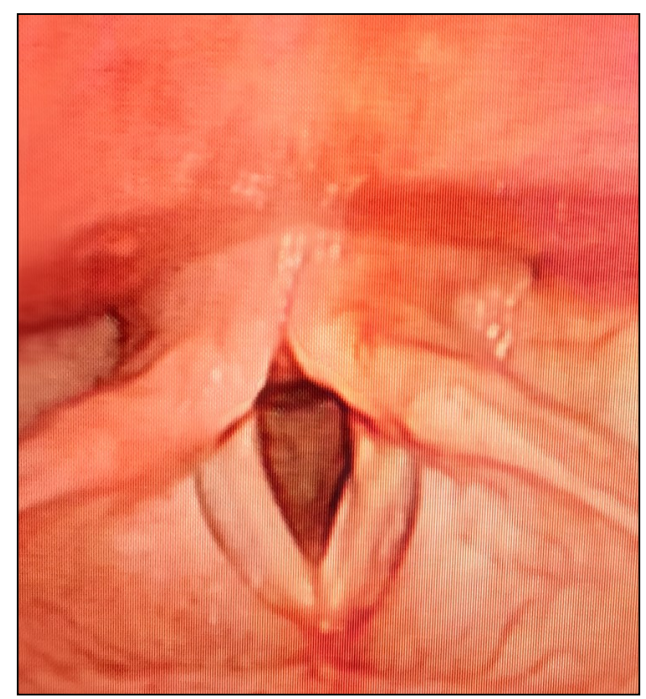

Figure 3: Postoperative endoscopic view of bilateral normal vocal cords. 


\section{DISCUSSION}

Adult laryngeal hemangiomas are rare benign vascular tumors that differ from infantile hemangiomas in terms of aetiopathogenesis, histopathology, natural history, and clinical presentations. ${ }^{5}$ The adult laryngeal hemangioma is scarce and vocal cord localization is even scarcer. ${ }^{5}$ The diagnosis was made primarily by history and physical examination were the fact that the presenting symptoms of patients depend on the location of the lesion. Symptoms may include dysphagia, hoarseness, and shortness of breath in adults, and stridor and respiratory distress in children.

In this present case, given the presentation history where he had hoarseness for one year, later presented with blood in sputum and difficulty of breathing, in addition of his age and social history with an associated physical examination that revealed an irregular red-purplish polypoidal lesion over the left vocal cord, we cannot be certain that it was a benign tumor without a tissue biopsy. It was reported that biopsy of typical laryngeal lesions is not essential, ${ }^{6}$ however, in this case, we found that a tissue biopsy is a must to exclude malignant neoplasm and it must be done cautiously due to respiratory and hemorrhagic risks.

Histopathologically, hemangiomas can be divided into three types; capillary, cavernous, and mixed type, cavernous being the commonest. ${ }^{7}$ It was reported by Mulliken and Glowacki that infantile hemangioma is characterized by endothelial cell proliferation in the rapid growth phase, followed by a spontaneous involution phase, whereas in adult hemangioma, it composed of dysplastic vessels with endothelial cells presenting a normal turnover. ${ }^{8}$ These adult forms are present at birth and can remain stable or progress during life. ${ }^{8}$ Adult hemangiomas are therefore a submucosal venous malformation. The mucosa may be intact or thickened and inflammatory. The highly suggestive bluish discoloration may be absent and vascular malformation may be situated underneath a smooth and perfectly pink mucosa.

Computer tomography (CT) scan and magnetic resonance imaging (MRI) have helped determine the type, size, and anatomical relation of hemangiomas. Chetty at al. demonstrated that contrasted CT scan aided the detection of subglottic hemangiomas and as well revealed a degree of airway narrowing. ${ }^{9}$ Alternatively, MRI may furnish excellent differentiation of muscle and fat, thereby it is useful in determining the depth of vascular malformation and delineating the tumor from normal tissue.

The combined use of imaging and laryngoscopy may expedite the diagnosis of extensive laryngeal hemangiomas, however, we found that tissue biopsy remains essential to form a definitive diagnosis. In this present case, our patient did not undergo any imaging study because the hemangioma was confined to only the glottic region with no extension to the adjacent structures on endoscopic examinations. Moreover, the first histopathological biopsy revealed benign features with no evidence of malignancy, hence radiological imaging seemed unnecessary.

Small hemangiomas may be treated conservatively, however large hemangiomas with obstructive symptoms frequently require treatment. To the authors' knowledge, there are no well-established treatment protocols for laryngeal hemangiomas due to the scarcity of cases being reported. The treatments suggested include ethanol or corticosteroid injections, surgical resection, cryosurgery, gold or radium implants, interferon treatment, and laser surgery. ${ }^{6,10}$

In the present case, the patient presented with acute symptoms, and as such an active intervention is warranted. He underwent endolaryngeal microsurgery using cold instruments and the mass was excised with minimal bleeding. The authors' technique of choice is simple superficial excision where the underlying uninvolved lamina propia is spared and epithelial loss is minimized. This technique is sufficient to remove a pedunculated mass resulting in minimal tissue defect from excision and at the same time provide minimal scarring to the vocal fold as there was no dissection done between layers of the vocal fold.

By using this technique the patient's voice may be preserved, however, a meticulous surgical technique and delicate micro laryngeal instruments are required. Besides, the use of cold instruments may nullify the risk of periphery tissue damage which may be caused by dissipated thermal energy and offset the inherent danger of potentially catastrophic airway fire. 


\section{CONCLUSION}

Adult laryngeal hemangiomas are rare and glottic origin is even rarer. Tissue biopsy is essential for cases where the natural history, clinical presentations, and examinations were suspicious of a malignant neoplasm. Surgical intervention is needed for adult cases with obstructive symptoms. Endolaryngeal microsurgery using cold instruments may be an option when the hemangioma is covered with thick mucosa with minimal risk of bleeding.

\section{REFERRENCES}

1. Killcline C, Frieden IJ. Infantile hemangioma: how common are they? A systematic review of the medical literature. Pediatr Dermatol 2008;25 (2):168-73.

2. Mulliken JB, Fishman SJ, Burrows PE. Vascular anomalities. Curr Probl Surg 2000;37(8):517-84.

3. Grant DG, Birchall MA, Bradley PJ. Surgery for benign tumors of the adult larynx. In : Remade M, Eckel HE, eds. Surgery of Larynx and Trachea. Berlin, Heidelberg: Springer, 2010:91-111.

4. Nor Azirah S, Shahrul Hitam, Nor Eyzawiah H, Mawaddah Azman, Maizaton Atmadini A, Nor Azian A. Glottic hemangioma: a rare entity in adult. Rawal Medical Journal 2017;42(1):130-132.

5. Prasad SC, Prasad KC, Bhat J. Vocal cord hemangioma. Med J Malaysia 2008;63(5):419-20.

6. Martins RH, Lima Neto AC, Semenzate G, Lapate R. Laryngeal hemangioma. Braz J Otorhinolaryngol 2006;72(4):574.

7. Yang GZ, Li J, Jin H. Giant mesenteric hemangioma of cavernous and venous mixed type: a rare case report. BMC Surg 2013;13:50.

8. Mulliken JB, Glowacki J. Hemangiomas and vascular malformations in infants and children: a classification based on endothelial characteristics. Plast Reconstr Surg 1982;69 (3):412-22.

9. Chetty A, Mischler E, Gregg D. Diagnosis of subglottic hemangioma by chest CT. Pediatr Pulmonol 1997;23:464-67.

10. Zheng JW, Zhou Q, Yang XJ, Wang YA, Fan XD, Zhou GY, et al.Treatment guideline for hemangiomas and vascular malformations of the head and neck. Head Neck 2010;32(8):1088-98. 